\title{
Shifted Normal Forms of Polynomial Matrices
}

\author{
Bernhard Beckermann \\ Laboratoire d'Analyse Numérique et d'Optimisation \\ UFR IEEA M3, USTL Flandres Artois \\ F-59655 Villeneuve d:Ascq CEDEX, France \\ bbecker@ano.univ-lille1.fr
}

\author{
George Labahm \\ Department of Computer Scicnce \\ University of Waterloo, Ontario, Canada \\ glabahn@daisy.uwaterloo.ca
}

\author{
Gilles Villard \\ LMC-IMAG, BP 53 \\ F-38041 Grenoble cedex 9 \\ Gilles.Villard@imag.fr
}

\begin{abstract}
In this paper we study the problem of transforming, via invertible column operations, a matrix polynomial into a varicty of shifted forms. Examples of forms covered in our framework include a column reduced form, a triangular form, a Hermite normal form or a Popov normal form along with their shifted counterparts.

By obtaining degree bounds for mimodular multipliers of shifted Popov forms we are able to cmbed the problem of computing a normal form into one of determining a shifted form of a minimal polynomial basis for an associated matrix polynomial. Shifted minimal polynomial bases can be computed via sigma bases [2,3] and in Popov form via Mahler systems [4]. The lattcr method gives a fraction-frec algorithm for computing matrix normal forms. Key words: Popov Form, Hermite Normal Form
\end{abstract}

\section{Introduction}

Matrix polynomial arithmetic is fundamental to many applications in scicnce and engineering. It is enconntered in linear systems theory [12], determining minimal partial realizations of matrix sequences [20] and solving linear diophantine cquations $[14,16]$. Not surprisingly, the arithmetic: of matrix polynomials has substantial differences to that found with scalar polynomials. Such fundamnntal operations as determining a degree, a leading coefficient, and normal and canonical forms [10] have numerous variations in the matrix case. For example, the degree of a scalar polynomial has equivalents such as row degrec, column degree, degree, degree of the determinant, MacMillan Degrec, and others, each with their own usefulness. Leading coefficients of a matrix polynomial can mean leading column or leading row matrices and are not neccssarily invertible, a problem when attempting division-like computations.

Permission to make digital or hard copies of all or part of this work for personal or classroom use is granted without. fee provided that copics are not macle or distributed for profit or commercial advantage, and that copies bear this notice and the full citation on the first page. To copy otherwise, to republish, to post on servers or to redistribute to lists, recuires prior specific permission and/or a fee. ISSAC: 99. Vancouver, British Columbia, Canada. (C) $1999 \mathrm{AC} M 1-58113-073-2$ $/ 99 / 07 \$ 5.00$
In order to use many analogous concepts from scalar polynomial arithmetic one often transforms a given matrix polynomial into an equivalent matrix polynomial having better properties for fundamental operations. Formally, two matrix polynomials $\mathbf{A}(z)$ and $\mathbf{B}(z)$ arc column equivalent if there exists a unimodular polynomial matrix $\mathbf{U}(z)$ such that $\mathbf{A}(z) \cdot \mathbf{U}(z)=\mathbf{B}(z)$. The matrix $\mathbf{U}(z)$ corresponds to a sequence of elementary column operations. For a given $\mathbf{A}(z)$ one has a number of equivalent forms that are useful for applications. These include column reduced forms, where the leading column cocfficiont matrix is nonsingular (a property useful for division algorithms), triangular forms (useful for solving systems of linear equations) and Hermite normal forms or Popov normal forms (useful for determining when two matrix polynomials are not equivalent).

In this paper we study the problem of computing normal forms for full column rank matrix polynomials. In particular wo are interested in computiug Popov and shifted Popov normal forms for such matrices. Ronghly speaking, a Popov normal form $[17,22]$ is a form having a "good" structure for leading coefficient matrices on both row and column sides. It has the important property that it always reduces column degrees of the input malrix. This is different from other forms such as the classical Hermite normal form which is an upper triangular matrix with additional degrec constraints with respect to diagonals but. which typically has degrees increased during the reduction to normal form.

The notion of a shifted form is basically one of altering the degree structures of the rows of a matrix and then computing forms of the resulting matrix. It is a simple process but very powerful. For example, the classical Hermite normal form can be obtained by determining a shifted Popov normal form for a shift determined from the degree structure of the input matrix polynomial (cf. Example 2.5). Shifted Popor Forms for square nonsingular matrix polvnomials were first introduced in [4] as a convenient normal form for describing the properties of Mahler systems. Mahler systems were used as a basic building block for recursively computing solutions to module bases for matrix rational approximation and matrix rational interpolation problems (sec also [1] in the case of matrix Padé systems). Tho (vecior) shift was uscful in this application for keeping track of a path of computation that allowed one to avoid singular situations in order to recursively compute along a path of closest nor- 
mal points to a singularity. A (scalar) shift has also been used by Beclen et al [7] in determining a reduced column form of a full column rank matrix polynomial by computing a minimal polynomial basis of a shifted stacked rectangular matrix. The use of a shift ensured that the nonsingular leading coefficient matrix be isolated in specific (in this case last) rows.

By obtaining degree bounds for unimodular multipliers of shifted Popov forms, we are able to cmbed the problem of conputing a normal form into one of determining a shifted form of a mininual polynomial basis for an associated matrix polynomial kernel. Shifted minimal polynomial bases can be computed via sigma bases $[2,3]$ and in Popov form via Mahler systems [4]. The last named algorithm has an important property: if the entries in the original matrix arc polynomials having coefficients from an integral domain (for example a matrix with entries from $\mathbf{Z}[z]$ or $\left.\mathbb{Q}\left[a_{1}, \ldots, a_{k}\right][z]\right)$ then it computes a minimal polynomial bases using only fraction-frce arithmetic.

The results in this paper are part of a larger research program: the efficient computation of matrix normal forms for arbitrary matrix polynomials. In particular we are interested in efficient fraction-free computation of such normal forms for nonsingular, singular and rectangular matrices. Our results give a first step in this direction. Additional results are available in the manuscript [6].

The remainder of the paper is organized as follows. Section 2 gives the basic definitions of shifted reduced and normal forms in the case of nonsingular square matrices while the next section looks at the equivalent problem for rectangular matrix polynomials of full column rank. Section 4 gives degree bounds on the (unique) unimodular multiplier. Section 5 shows how to embed the problem of computing a shifted Popov Normal Form and the associated unimodular multiplier into one of computing a minimal polynomial basis in normal form. This allows shifted normal forms to be computed using the algorithm of [4]. The last section includes a conclusion along with a discussion of future research directions.

\section{Shifted Popov Forms of Nonsingular Matrices}

In this section we give some of the basic definitions and properties required for the remainder of the paper for the case where the input matrix polynomial is square and nonsingular. Various shifted forms are introduced along with the concept of shifted column reduction. We remark that for any multi-index $\vec{a}$ (i.e. a vector of integers) we denote by $|\vec{a}|$ the sum of its components, max $\vec{a}$ its maximum component and perm $(\vec{a})$ a permutation of the components. In addition the multi-index $\vec{e}$ denotes the vector $(1, \ldots, 1)$.

Definition 2.1 ( $\vec{a}$-Shifted forms, square matrices)

An $m \times m$ matrix polynomial $\mathbf{T}(z) \in \mathbb{Q}^{m \times m}[z]$ is $\vec{a}$-column reduced with $\vec{a}$ column degree $\vec{\alpha}$ - if there exists a multiindex $\vec{\alpha}$ such that

$$
\begin{aligned}
z^{-\vec{a}} \cdot \mathbf{T}(z) \cdot z^{\vec{a}-\vec{\alpha}}= & \mathbf{T}^{\prime}+\mathcal{O}\left(z^{-1}\right): \rightarrow \infty \\
& \text { with } \mathbf{T}^{\prime} \in \mathbb{Q}^{m * m} \text { nonsingular. }
\end{aligned}
$$

If this condition holds with $\mathbf{T}^{\prime}$ nonsingular and upper triangrular then $\mathbf{T}(z)$ is said to be in $\vec{a}$ quasi Popov form. When it satisfies the additional normalization degree and leading coefficient constraint

$$
z^{-i} \cdot \mathbf{T}(z)=\mathbf{I}_{m}+\mathcal{O}\left(z^{-1}\right)_{i \rightarrow \infty} .
$$

then $T(z)$ is said to be in $\vec{a}$ Popov normal form.

Remark 2.2 Propertics (1) and (2) are invariant under adding a constant to all components of $\vec{a}$. This allows us, in particular; lo exlerud our definitions to Laurent matrix polynomials where needed.

Up to a (unique) permutation of columns, Definition 2.1 gines the classical Popov normal form [12. Subsection 6.7.2, p.481] in the case $\vec{a}=\overrightarrow{0}$. When the form is used in a matrix fraction description or as a minimal polynomial basis, the degree $\vec{\alpha}$ are referred to as the vector of controllability or Kronecker indices.

For any tuo column equivalent and $\vec{a}$-column reduced matrices, the corresponding vectors $\vec{\alpha}-\vec{a}$ as in (1) coincide up to permutation [12, Lemma 6.3-14, p.388]. Also, for any matrix polynomial $\mathbf{A}(z)$ column equivalent to $\mathbf{T}(z)$ as in (1),

$$
|\vec{\alpha}|=\operatorname{deg} \operatorname{det} \mathbf{T}(z)=\operatorname{deg} \operatorname{det} \mathbf{A}(z) .
$$

is un invariant.

It is known $[12, \S 6.7 .2, p .484]$ that any square nonsingular matrix polynomial may be transformed to Popov normal form by multiplication on the right by a unimodular matrix polynomial, and that the form is unique. A similar statement is also true for an $\vec{a}$-Popov form.

Lemma 2.3 For a nonsingular matrix polynomial $\mathbf{A}(z)$ and a multi-index $\vec{a}$, set $\mathbf{A}(z)^{\#}=z^{-\vec{a}} \cdot \mathbf{A}(z)$. Let $\mathbf{T}(z)^{\#}=$ $\mathbf{A}(z)^{\#} \cdot \mathbf{U}(z)$ be the $\overrightarrow{0}$-.Popov form with degree $\vec{\alpha}^{\#}$ of this resulting Laurent matrix polynomial $\mathbf{A}(z)^{\#}$. Then $\mathbf{T}(z)=$ $z^{\vec{a}} \cdot \mathbf{T}(z)^{\#}=\mathbf{A}(z) \cdot \mathbf{U}(z)$ is an $\vec{a}-$ Popov form of $\mathbf{A}(z)$ with degree $\vec{\alpha}=\vec{\alpha}^{\#}+\vec{a}$. Conversely, for any $\vec{a}$-Popov form $\mathbf{T}(z)$ of $\mathbf{A}(z)$ we have $\mathbf{T}(z)^{\#}=z^{-\vec{a}} \cdot \mathbf{T}(z)$.

Lemma 2.3 says that it is possible to consider only $\overrightarrow{0}-$ Popov forms. However, the introduction of an additional parameter $\vec{a}$ is convenient for a number of reasons. It appears naturally in the context of the approximation problems studied in [4], and was the primary purpose for introducing this form. Our vector shift can also be used to simplify the Matrix Eucliclean algorithm of [8]. Indeed their six reduction steps can be viewed as moving from one shifted Popov form to a new shifted Popor form with a reduced shift. In the case of column-reduction a shift (in this case a scalar shift of cortain componcnts) was used as a tool in the algorithm of $[\bar{l}]$ for constructing a column reduced polynomial matrix for a matrix polynomial of full column rank.

A vector shift is also very useful in that it allows one to describe a number of other important matrix normal forms. For example, triangular forms and the Hermite normal form $[15, \S 22$, p.32] are obtained with shifts as follows.

Lemma 2.4 Let $\mathbf{A}(z)$ be a matrix polynomial and $\mathbf{T}(z)$ be one of its $\vec{a}$-quasi Popov forms with $\vec{a}$-degree $\vec{\alpha}$. If $\vec{a}$ and $\vec{\alpha}$ satisfy

$$
\vec{a}_{i}-\vec{a}_{i} \geq \vec{\alpha}_{j}, \text { for } i>j,
$$

then $\mathbf{T}(z)$ is upper triangular. Furthermore the $\vec{a}$-Popov form of $\mathbf{A}(z)$ coincides with its Hermite normal form.

Proof. Let us show that $\mathbf{T}(z)_{i, j}=0$ for $i>j$. By Definition 2.1, $\mathbf{T}^{\prime}$ is upper triangular thus for $i>j$, $-\vec{a}_{i}+\operatorname{deg} \mathbf{T}(z)_{i, j}+\vec{a}_{j}-\vec{a}_{j}<0$ and if (4) is true then 
$\operatorname{deg} \mathbf{T}(z)_{i, j}<0$ and $\mathbf{T}(z)$ is upper triangular. Also condition (2) implies that the degree of a diagonal entry is strictly larger than the degree of the other entries of this row and the $\vec{a}$-Popor form is the Hermite normal form.

The condition $\mathbf{T}^{\prime}$ upper triangular in Definition 2.1 plays a minor role in the entire triangularization of $\mathbf{A}(z)$. Indecd the upper triangularization may be ensured using only a column reduced form with a slightly different shift. If $\mathbf{T}(z)$ is $\vec{a}$-column reduced with $\vec{a}_{j}-\vec{a}_{i}>\vec{\alpha}_{j}$, for $i>j$, then $\mathbf{T}(z)$ is upper triangular.

Even if for any given matrix $\mathbf{A}(z)$, the degrees $\vec{\alpha}_{j}$ are not known in advance, one may always use Lemma 2.4 and shifted forms to compute triangular forms. Indeed, the degrees are bounded by (3) thus choosing $w \geq \operatorname{deg} \operatorname{det} \mathbf{A}(z)$ and as shift vector $\vec{a}=[(m-1) w, \ldots, u, 0]$, condition $(4)$ is satisficd.

Example 2.5 Let $\mathbf{A}(z)$ be the folloning $\overrightarrow{0}$-column reduced matrix:

$$
\mathbf{A}(z)=\left[\begin{array}{ccc}
z^{2}-1 & z+1 & 2 \\
0 & -z & z-2 \\
z+2 & 0 & z+1
\end{array}\right]
$$

From the sum of the column degrees one may take $w=4$ as an upper bound on the degres of the determinant. If $\vec{a}=$ $[8,4.0]$ then an $\vec{a}$-quasi Popov form of $\mathbf{A}(z)$ is

$$
\left[\begin{array}{ccc}
z^{4}+z^{2}-z+4 & -z^{3}+4 z+5 & z^{4}-4 z^{3}+4 z^{2}+15 z \\
0 & -4 & -8 z \\
0 & 0 & 8
\end{array}\right],
$$

which in fact is $[4,1,0]$-reduced with degree $\vec{\alpha}=[4,0,0] . B y$ Lemma 2.4, with the same shifts, the $\vec{a}$-Popov form of $\mathbf{A}(z)$ :

$$
\left[\begin{array}{ccc}
z^{4}-4 z^{2}-z+1 & \frac{1}{4} z^{3}-z-\frac{5}{4} & \frac{1}{2} z^{3}+z^{2} \mid z-\frac{3}{2} \\
0 & 1 & 0 \\
0 & 0 & 1
\end{array}\right]
$$

is also its Hermite normal form.

This application of shifted forms of matrix polynomial, is very similar to a well known application of integer lattice basis reduction. Given an integer matrix $\mathbf{A}$, it is shown in [18, p 74] that the reduction of a well chosen lattice deduced from A, gives the integer Hermite form.

\section{Shifted Forms of Full Column Rank Matrices}

In order to include in our framework such applications as the extended gcd problem (cf. Example 3.4), determination of matrix structures [21] (cf. Example 3.5) and normalization of module bases, we extend the results of the previous section to full column rank polynomial matrices. We introduce shifts in the classical treatment for column reduced forms $[17,12$, p481] and, as done: in [13] in the Hermite case, we ensure the normalization of leading matrices by considering columm echelon forms.

Given a matrix polynomial $\mathbf{A}(z)$, we denote its olements by $\mathbf{A}(z)_{i, j}$. Furthermore, given lists $I, J$ of increasing row $/$ column indices of $\mathbf{A}(z)$, we denote by $\mathbf{A}(z)_{1,1}$ the corresponding submatrix of $\mathbf{A}(z)$. Also, for $\mathbf{A}(z)_{I, *}$ (and $\left.\mathbf{A}(z)_{*, J}\right)$ we just extract rows with index in $I$ (and columns with index in $J$, respectively).
Definition 3.1 (Column echelon matrices) $A$ full column rank scalar matrix $\mathbf{T}^{\prime} \in \mathbb{Q}^{m \times n}$ is in upper echelon form with pivot set $I=\left(i_{1}, \ldots, i_{n}\right)$ if $1 \leq i_{1}<i_{2}<\ldots<i_{n} \leq m$, $\mathbf{T}_{i, j}^{\prime}=0$ for $i>i_{j}$, and $\mathbf{T}_{i_{j, j}}^{\prime} \neq 0, j=1, \ldots, n$.

Any full column rank scalar matrix may be transformed by column operations to upper echelon form and the corresponding pivot set is unique. The (row) pivot set of any matrix is thus well defined as the pivot set of its (column) upper echelon forms. In addition we have:

Lemma 3.2 The pivot set $I$ is maximal for det $\mathbf{T}_{L_{*}}^{\prime}$ being nonzero, that is, if $I^{\prime}=\left(i_{1}^{\prime}, \ldots, i_{\ell}^{\prime}, i_{f+1}^{\prime}, \ldots, i_{n}^{\prime}\right)$ with $i_{\ell}^{\prime}>i_{k}$ and $i_{k}^{\prime} \geq i_{k}, \ell+1 \leq k \leq n$, then $\operatorname{det} \mathbf{T}_{I^{\prime} .=}^{\prime}=0$.

Proof. By Definition 3.1, the rows of indices $i_{f}^{\prime}, \ldots, i_{n}^{\prime}$ cannot be linearly independent since their first $\ell$ entries are zero.

Dofinition 2.1 is generalized for non-square matrices.

Definition 3.3 A matrix polynomial $\mathbf{T}(z) \in \mathbb{Q}^{m \times n}[z]$ is called $\vec{a}$-column reduced with degree $\vec{\alpha}$ if there exists a full column rank scalar matrix $\mathbf{T}^{\prime}$ with pivot set I satisfying

$$
z^{-\vec{a}} \cdot \mathbf{T}(z) \cdot z^{\vec{n},-\vec{\alpha}}=\mathbf{T}^{\prime}+\mathcal{O}\left(z^{-1}\right)_{i \rightarrow \infty} .
$$

If $\mathbf{T}^{\prime}$ is in upper echelon form then $\mathbf{T}(z)$ is said to be in $\vec{a}-$ quasi Popov form. If it satisfies the additional normalization degree and leading coefficient constraint

$$
z^{-\vec{x}} \cdot \mathbf{T}(z)_{I, *}=\mathbf{I}_{n}+\mathcal{O}\left(z^{-1}\right)_{z \rightarrow \infty}
$$

then $\mathbf{T}(\approx)$ is said to be in $\vec{a}$-Popov normal form.

By extension of Definition 3.1, we will refer to the set $I=\left(i_{1}, \ldots, i_{n}\right)$ of Definition 3.3 as the pivot set of an $\vec{a}-$ columm reduced matrix $\mathbf{T}(z)$. If $\mathbf{T}(z)$ in addition is in $\vec{a}-$ quasi Popov form then its entry $\mathbf{T}(z)_{i_{j}, j}$ - referred to as the $j$ th pivot clcment - has precise degrec $\vec{\alpha}_{j}$. Notice that the statenent of Lemma 2.3 also holds in the rectangular case. In particular, $\mathbf{A}(z)$ is $\vec{a}$-colnmm reduced if and only if $z^{-\vec{a}} \cdot \mathbf{A}(z)$ is $\overrightarrow{0}$-column reduced. Non normal shifted forms have becn defined in [20] with a slightly different definition of the $\vec{a}$-degree.

The next example gives another illustration of the link between shifted forms of polynomial matrices and the integer case. We use the fact that a shift vector with only one nonzero entry may be used to "select" a given row in a matrix. As shown in [11, Algorithm 2] for integers, this is useful in solving the extended ged problem.

Example 3.4 To compute the gcd $c(z)$ of $a(z)=z^{2}-4 z+3$ and $b(z)=z^{3}-7 z^{2}+14 z-8$ together with a corresponding multiplier $\mathbf{U}(z)$ such that $[a(z) b(z)] \cdot \mathbf{U}(z)=[c(z) 0]$, one may compute a shifted form equivalent to

$$
\mathbf{A}(z)=\left[\begin{array}{cc}
z^{2}-4 z+3 & z^{3}-7 z^{2}+14 z-8 \\
1 & 0 \\
0 & 1
\end{array}\right]
$$

This matrix: is buill using the identity matrix, to keep track of the column operations that arc performed and that will give $\mathbf{U}(z)$. $A \overrightarrow{0}$-column reduced form of $\mathbf{A}(z)$ is

$$
\left[\begin{array}{cc}
z^{2}-4 z+3 & -z+1 \\
1 & -z+3 \\
0 & 1
\end{array}\right]
$$


but does not provide the target result. One could verify here that a $[-2,0,0]$-roduced form:

$$
\left[\begin{array}{cc}
-z+1 & 0 \\
-z+3 & -z^{2}+6 z-8 \\
1 & z-3
\end{array}\right]
$$

leads to $(z)$ and to a possible $\mathbf{U}(z)$.

The generalization of Remark 2.2 and in particular of identity (3) relies on classical tools from linear system theory. For a matrix polynomial $\mathbf{A}(z)$ of full column rank we define the Minor degree - denoted by Minor-deg $\mathbf{A}(z)$ - as the maximum of the degrees of the determinants of $n \times n$ submatrices of $\mathbf{A}(z)$ (seo [12, Fq. (34), p.4j4]). This can be naturally extended to Laurent matrix polvnomials. Sotting $\vec{\alpha}^{*}=\operatorname{cdeg}\left(z^{-\vec{a}} \mathbf{A}(z)\right)$ where cdeg denotes the unshifted column degree, it is well-known that

$$
\left|\vec{\alpha}^{*}\right| \geq \operatorname{Minor-dleg}\left(z^{-\vec{a}} \cdot \mathbf{A}(z)\right)
$$

with equility iff $\mathbf{A}(z)$ is $\vec{a}-$ column reduced $[12$, $\$ 6.3 .2$, p.384]. In this latter case, with pivot set $I$. Lomma 3.2 provides the additional information

$$
\begin{aligned}
& \text { Minor-deg }\left(z^{-\vec{u}} \mathbf{A}(z)\right)=\operatorname{deg} \operatorname{det}\left(z^{-\ddot{u}_{I}} \mathbf{A}(z)_{r, *}\right) \\
& =\left|\overrightarrow{\hat{\alpha}}^{2}\right| \\
& >\operatorname{deg} \operatorname{det}\left(z^{\cdot} \ddot{ }_{I^{\prime}} \mathbf{A}(z)_{I^{\prime}, \ldots}\right)
\end{aligned}
$$

where $I^{\prime}$ is any list as in the lemma.

Example 3.5 The $\overrightarrow{0}$-Popow normal of

$$
\mathbf{A}(z)=\left[\begin{array}{ccc}
z^{2} & -z^{2}+1 & z^{4}-z^{2}+z \\
z-1 & -z+2 & z^{3}-2 z^{2} \\
z & 0 & 1 \\
1 & -1 & z^{2} \\
z+1 & -z-2 & z^{3}+3 z^{2}
\end{array}\right]
$$

has pinot set $I=(1,3,5)$ and vector degree $\vec{\alpha}=[2,1,2]$ :

$$
\mathbf{T}(z)=\left[\begin{array}{ccc}
\underline{z^{2}}-1 & 1 & z \\
z-2 & 1 & 0 \\
0 & \underline{z} & 1 \\
1 & 0 & 0 \\
z+2 & -1 & \underline{z}
\end{array}\right] .
$$

The form reveals that $\mathbf{A}(z)$ has Minor degree $|\overrightarrow{\boldsymbol{n}}|=5$. Herc, the degress are also called the minimal indices of the module generated by the columns of $\mathbf{A}(z)$ (sce also Definition 5.1) [9]. With $\vec{a}=[0,0,0,0,3]$, the shifted form.

$$
\mathbf{T}^{\vec{a}}(z)=\left[\begin{array}{ccc}
\underline{z} & 0 & 1 \\
0 & \underline{z}-1 & 1 \\
1 & 0 & \underline{z} \\
0 & 1 & 0 \\
z^{2} & -z^{3}+z+1 & -1
\end{array}\right]
$$

has pivol set $I=(1,2,3)$ and vector degree $\vec{\alpha}=[1,1,1]$. Now $|\vec{a}|=3$ is the Minor degree of the first 4 rows of $\mathbf{A}(\approx)$.
Theorem 3.7 below shows that any polynomial matrix can be transformed to a unique $\vec{a}$-Popov form by multiplication on the right with some unimodular matrix. We first state a lemma which gives a useful property of column reduced matrices (see [12, Theorem 6.3-13, p387]).

\section{Lemma 3.6 (Predictable-Degree Property)}

Let $\mathbf{B}(z)$ with coleg $z^{-\dot{b}} \cdot \mathbf{B}(z)=\vec{\beta}^{*}$, be a $\vec{b}$-column reduced matria polynomial. If $\mathbf{P}(z)$ and $\mathbf{C}(z)$ are tuo matrict polynomials such that $\mathbf{B}(z) \mathbf{P}(z)=\mathbf{C}(z)$ with cdeg $z^{-\vec{b}} \cdot \mathbf{C}(z)=\vec{\gamma}^{*}$ then $\operatorname{deg} \mathbf{P}(z)_{i, j} \leq \vec{\gamma}_{j}^{*}-\vec{\beta}_{i}^{*}$.

\section{Theorem 3.7 ( $\vec{a}$-Popov form)}

Any matrix $\mathbf{A}(\xi) \in \mathbb{Q}^{m \times n}[\approx]$ of full column rank is equivulent to a unique matrix $\mathbf{T}(z)$ in $\vec{a}-P^{3}$ opov normal form.

Proof: The existence of a columm reduced form $\mathbf{B}(z)$ equivalent to $\mathbf{A}(z)$ is a classical fact, see $[12, p 386]$ for instance. The same applies dircctly to shifted forms. Following (j) let $\mathbf{B}^{\prime}$ be the corresponding leading scalar matrix. We denote by $\vec{\beta}^{\star}$ the unshifted degrer:

$$
\begin{aligned}
z^{-\vec{a}} \cdot \mathbf{A}(z) \cdot \mathbf{U}(z) \cdot z^{-\vec{\beta}^{*}} & =z^{-\vec{a}} \cdot \mathbf{B}(z) \cdot z^{-\vec{\beta}^{*}} \\
& =\mathbf{B}^{\prime}+\mathcal{O}\left(z^{-1}\right)_{z \rightarrow \infty}
\end{aligned}
$$

and let $I$ be the pivot set of $\mathbf{B}^{\prime}$. If $\mathbf{T}(\dot{z})_{l, x}=\mathbf{B}(z)_{I, *} \mathbf{V}(z)$ is in $\vec{a}_{I}$-Popoy form (Definition 2.1 in the monsingular case) then $\mathbf{T}(z)=\mathbf{A}(z) \cdot \mathbf{U}(z) \cdot \mathbf{V}(z)$ is in $\vec{a}$-Popov form. Indeed, by Remark 2.2 , the components of the unshifted degree $\vec{\alpha}^{\star}$ of $z^{-\vec{u}_{I}} \cdot \mathbf{T}(z)_{I, *}$ are given by a permutation of the components of $\vec{\beta}^{*}: \vec{\alpha}^{*}=$ perm $\left(\vec{\beta}^{*}\right)$. Lemma 3.6 implies that $\operatorname{deg}[\mathbf{V}(z)]_{i, j} \leq \vec{\alpha}_{j}^{*}-\vec{\beta}_{i}^{*}$. Thus all the entries in the $j$-th column of $z^{-\vec{a}} \cdot \mathbf{T}(z)$ have degrees bounded by $\vec{\alpha}_{j}^{*}$. Using the second identity of (7) we have Minor-deg $\left(z^{-\bar{a}} \mathbf{T}(z)\right)=$ Minor-deg $\left(z^{-\vec{n}} \mathbf{B}(z)\right)$ thus the two matrices must have the same pivot set $I$, otherwise the inequality in ( 7 ) would be violated. For the same reason, it follows that bolow a pivot entry in $z^{-\ddot{a}} \mathbf{T}(z)$ the degrees must be strictly less than $\vec{\alpha}_{j}^{*}$, this establishes the existence of the form.

By $(7)$, as used above, any two $\vec{a}$-column reduced matrices that are unimodular column equivalent have the same pivot set. From the nniqueness of the form in the nonsingular case, if $T^{(1)}(z)$ and $T^{(2)}(z)$ are two $\vec{a}$-Popoy forms of $\mathbf{A}(z)$ then $\mathbf{T}^{(1)}(z)_{I, *}=\mathbf{T}^{(2)}(z)_{l . *}$. This implies that the two corresponding transformation matrices are the same and that $\mathbf{T}^{(1)}(z)=\mathbf{T}^{(2)}(z)$.

Wo have seen in the preceding proof that, for any two column equivalent and $\vec{a}$-column reduced matrices, the pivot sets coincide. Nevertheless, by $(\bar{T})$, the pivot set will in general depend on $\vec{a}$. For instance, gencralizing Lemma 2.4 to the full rank case we may define Hermite shifts $\vec{a}$ by

$$
\vec{a}_{i_{j}}-\vec{a}_{i} \geq \vec{\alpha}_{j}, \text { for } i>i_{j} .
$$

In such cascs, $I$ will consist of the largest row indices such that $\mathbf{A}(z)_{l . \Omega}$ is nonsingular.

\section{Degree bounds for multipliers}

In this section we give degree bounds for the unimodular multiplier $\mathbf{U}(z)$ used to transform a given matrix polynomial $\mathbf{A}(\varepsilon)$ into an $\vec{a}-$ Popov form (or sorne similar form) $\mathbf{T}(z)$. The 
bounds will be used in the next section to embed the shifted Popov problem into one of computing a certain basis of the kernel of an associated matrix polynomial, a computation that can be efficiently done in computer algebra systems.

Our degree estimates will be given in tcrms of a frec pa rameter $\vec{c}$ which may be chosen in order to reflect particular properties of the input $\mathbf{A}(z)$ (e.g., in the case where $\mathbf{A}(z)$ is $\vec{c}$-column reduced). In the first part of the next theoren our estimates are formulated in terms of the inputs $\mathbf{A}(z), \vec{a}, \vec{c}$, and of the invariants $\mathbf{T}(z), \vec{\alpha}, I$. The aim of the second part is to estimate the invariants in terms of the input.

'Theorem 4.1 (Degree bounds for Multiplier)

Let $\mathbf{A}(z) \cdot \mathbf{U}(z)=\mathbf{T}(z)$ uith $\mathbf{U}(z)$ unimodular and $\mathbf{T}(z)$ being $\vec{a}$-column reduced with pivot set $I$ and degree $\vec{\alpha}$. Furthermore, for some arbitrary multi-index $\vec{c}$, let ${ }^{1}$

$$
\vec{\gamma}^{-\vec{c}}=\operatorname{cdeg}\left(z^{-\vec{c}} \cdot \mathbf{A}(z)\right): \quad \vec{\tau}^{* \vec{c}}=\operatorname{cdeg}\left(z^{-\vec{c}} \cdot \mathbf{T}(z)\right),
$$

and define $\Delta^{\vec{c}}:=\left|\vec{\gamma}^{* \vec{c}}\right|+\left|\vec{c}_{I}\right|-|\vec{\alpha}|$. Then

$$
\operatorname{deg} \mathbf{U}(z)_{k, j} \leq \vec{\tau}_{j}^{* \vec{c}}-\vec{\gamma}_{k}^{* \vec{c}}+\Delta^{\vec{c}} ; \quad j, k=1, \ldots, n .
$$

Also

$$
0 \leq- \text { Minor-deg }\left(z^{-\vec{c}} \cdot \mathbf{A}(z) \cdot z^{-\vec{\gamma}^{* \vec{c}}}\right) \leq \Delta^{\vec{c}} \leq\left|\vec{\gamma}^{* \vec{c}}\right||| \vec{c}_{T} \mid
$$

and $\Delta^{\vec{c}}=0$ iff $\mathbf{A}(z)$ is $\vec{c}-$ column reduced. In addition,

$$
\begin{aligned}
& \vec{\tau}^{* \vec{c}} \leq \vec{\alpha}-\vec{a}_{I}+\vec{e} \cdot \max [\vec{a}-\vec{c}], \\
& \vec{\tau}^{* \vec{a}}=\vec{\alpha}-\vec{a}_{l} \leq \operatorname{perm}\left(\vec{\gamma}^{* \vec{a}}\right) .
\end{aligned}
$$

Before giving a proof of Theorem 4.1 we give some cxamples which illustrate the sharpness of $(9)$ for different choices of $\vec{c}$. In the examples $\mathbf{A}(z)$ will always be nonsingular so $m=n$ and $I=(1, \ldots, m)$. Also, we will just compare the choices $\vec{c}=\vec{a} \geq \overrightarrow{0}$ and $\vec{c}=\overrightarrow{0}$. If $\vec{\gamma}^{+\overrightarrow{0}}=\operatorname{cdeg} \mathbf{A}(z)$ and $\vec{\gamma}^{* \vec{a}}=\operatorname{cdeg}\left(z^{-a} \mathbf{A}(z)\right)$ are of the same magnitude, then $\Delta^{\overrightarrow{0}} \approx \Delta^{\vec{a}}-|\vec{a}| \leq \Delta^{\vec{\imath}}-\max [\vec{a}]$, and from (9) and (11) we see that the choice $\vec{c}=\overrightarrow{0}$ leads to tighter bounds (compare with $\mathbf{A}^{(3)}(z)$ below). If the shift is relevant for the degree bounds, typically when $\Delta^{\vec{a}} \leq \Delta^{\overrightarrow{0}}$ (see matrix $\mathbf{A}^{(2)}(z)$ ), then the choice $\vec{c}=\vec{a}$ will be more appropriate. However, the optimal choice of the paranieter $\vec{c}$ remains an open problem.

Example 4.2 Given the shift $\vec{a}=[0,6,9]$, we have $\vec{\gamma}^{* 0}=$ $\vec{\gamma}^{* \vec{a}}+\vec{a}=[8,3,1]$ (and thus $\Delta^{\vec{a}}=\Delta^{\overrightarrow{0}}$ ) for the matrix

$$
\mathbf{A}^{(1)}(z)=\left[\begin{array}{ccc}
2 z^{8} & 0 & 0 \\
z & z^{3}+z & 0 \\
z+1 & z+1 & 2 z
\end{array}\right]
$$

which is $\vec{a}$-column reduced. The transformation matrix for the $\vec{a}$-Popov form is constant, and the two estimates for $\vec{c} \in$ $\{\overrightarrow{0}, \vec{a}\}$ give the exact degree 0 for most of its coefficients.

For the shift $\vec{a}=[7,2,0]$ on the matrix

$$
\mathbf{A}^{(2)}(z)=\left[\begin{array}{ccc}
2 z^{10}+z & z^{8}-1 & z^{11}+1 \\
z^{5}+1 & 2 z^{3}-1 & z^{4}+1 \\
-z^{2} & 2 & 2 z
\end{array}\right]
$$

\footnotetext{
'As seen in the proof below, estimates $(9)$ and $(12)$ remain valid if insteac of $(8)$ we only assume that $z^{*} \vec{c}>\operatorname{cdeg}\left(z^{-\vec{c}_{r}} \cdot \mathbf{A}(z)_{1,+}\right)$ and $\vec{\tau}^{*} \vec{c} \geq \operatorname{cdeg}\left(z^{-\vec{c}_{I}} \cdot \mathbf{T}(z)_{1, *}\right)$.
}

one gets $\vec{\gamma}^{* \vec{a}}=\vec{a}+[10,3,4]$ and $\vec{\gamma}^{* \overrightarrow{0}}=[10,8,11]$. The $\vec{a}$ Popov form of $\mathbf{A}^{(2)}(z)$ has the degree vector $\vec{\alpha}=[10,3,3]$. With $\Delta^{\vec{a}}=1$ and $\Delta^{\overrightarrow{0}}=13>\Delta^{\vec{\imath}}$, the bound for $\vec{c}=\vec{a}$ is precise and gives the exact maximum degree 3 for the entries of the transformation, when the other one is pessimistic and gives a maximum degree 15 .

The $[10,3,0]-$ Popov form of

$$
\mathbf{A}^{(3)}(z)=\left[\begin{array}{ccc}
2 z+1 & 2 z^{5}+z^{4}+2 z^{3}+2 z & 2 z^{2}+2 \\
2 z+1 & z^{5}+z^{3}+2 z^{2}+1 & z^{2}+1 \\
z+1 & z^{5}+2 z^{4}+2 z^{2}+z & 2 z^{2}+z
\end{array}\right]
$$

is in upper triangular Hermite form with $\vec{\alpha}=[8,0,0]$. In this case $\Delta^{\vec{a}}=21>\Delta^{\overrightarrow{0}}=0$. The entries of the transformation $\mathbf{U}(z)$ have degree at most 7 and all the degrees are exactly predicted by the choice $\vec{c}=\overrightarrow{0}$. On the other hand the bound for $\vec{c}=\vec{a}$ gives a maximum degree 12 .

Proof of Theorem 4.1: For the remainder of the proof we fix $\vec{c}$ and use the shorter notation $\vec{\gamma}=\vec{\gamma}^{* \vec{e}}, \vec{\tau}=\vec{\tau}^{\times \vec{c}}$. For a proof of $(9)$, we recall that $\operatorname{deg} \operatorname{det} \mathbf{A}(z)_{I^{*}}=|\vec{\alpha}|$. Since $\mathbf{A}(z)_{i, j} \leq \vec{c}_{i}-\vec{\gamma}_{, j}$, we get

$$
z^{\vec{\gamma}} \cdot\left(\operatorname{adj} \mathbf{A}(z)_{I,-)}\right) \cdot z^{\vec{c}_{I}}=\mathcal{O}\left(z^{|\vec{\gamma}|+\left|\vec{c}_{I}\right|}\right) .
$$

Therefore, by applying (8) and Cramer's rule, the quantity $z^{\vec{f}} \cdot \mathbf{U}(z) \cdot z^{-\vec{r}}$ reduces to

$$
\frac{z^{\ddot{\gamma}} \cdot \operatorname{adj} \mathbf{A}(z)_{I, *} \cdot z^{\overrightarrow{c_{I}}}}{\operatorname{det} \mathbf{A}(z)_{I, *}} \cdot z^{-\vec{c}_{I}} \cdot \mathbf{T}(z)_{I, *} \cdot z^{-\vec{\tau}}=\mathcal{O}\left(z^{\Delta}\right)
$$

giving equation (9).

In order to show (10), recall first that $z^{-\vec{c}} \cdot \mathbf{A}(z) \cdot z^{-\bar{\gamma}}$ is a polynomial in $1 / z$ by the definition of $\vec{\gamma}$. Consequently,

$$
\begin{aligned}
0 & \geq \text { Minor-deg }\left(z^{-\vec{r}} \cdot \mathbf{A}(z) \cdot z^{-\vec{\gamma}}\right) \\
& \geq \operatorname{Minor-deg}\left(z^{-\vec{c}_{I}} \cdot \mathbf{A}(z)_{I, \times} \cdot z^{-\vec{\gamma}}\right),
\end{aligned}
$$

the latiter quantity being equal to $-\Delta^{\vec{c}}$. It remains to discuss the case $\Delta^{\vec{c}}=0$. By (7) and the above inequalities, $\Delta^{\vec{c}}=0$ is equivalent to the facts that $\vec{\gamma}$ is the colurnn degree both of $z^{-\vec{c}_{1}} \cdot \mathbf{A}(z)_{I, *}$ and $z^{-\vec{c}} \cdot \mathbf{A}(z)$, and that both matrices are column reduced, as claimed in (10).

Assertion (11) and the first part of (12) follow immedialely from the definition of $\vec{\tau}$. In order to show the final estimate for $\vec{c}=\vec{a}$, we introduce the unimodular matrix $\mathrm{V}(z)=$ $\mathbf{U}(z)^{-1}$. Since $z^{-\vec{a}} \mathbf{A}(z)=z^{-\vec{a}} \mathbf{T}(z) \cdot \mathbf{V}(z)$ and $z^{-\vec{a}} \mathbf{T}(z)$ is $\overrightarrow{0}$-column reduced, it follows from the Predictable-Degree Property (Lemma 3.6) and (8) that $z^{\vec{\tau}} \cdot \mathrm{V}(z) \cdot z^{-\vec{\gamma}}=\mathcal{O}\left(z^{0}\right)$. Since $V(z)$ is nonsingular, we find some permutation $\rho$ such that $\mathbf{V}(z)_{j, \rho(j)} \neq 0$, leading to $(12)$.

Remark 4.3 Recall that $\vec{\alpha}_{j}$ is the (a priori unknown) degree of a pivot element of the normal form, in particular it is nonnegative. Thus, in terms of the input data, combining (9) with (11) gives the weaker degree bound

$$
\operatorname{deg} \mathbf{U}(z)_{k, j} \leq\left|\vec{c}_{1}\right|-\vec{a}_{i_{j}}+\left|\vec{\gamma}^{* \vec{c}}\right|-\vec{\gamma}_{k}^{* \vec{c}}+\max [\vec{a}-\vec{c}] .
$$

In particular, in terms of $d=\operatorname{deg} \mathbf{A}(z)$, we obtain in the unshifted Popov case $(\vec{a}=\vec{c}=\overrightarrow{0})$

$$
\operatorname{deg} \mathbf{U}(z) \leq(n-1) \cdot d, \quad \operatorname{deg} \mathbf{T}(z) \leq d .
$$


In contrast, in the square Hermite case ( $m=n, \vec{c}=\overrightarrow{0}$, $\vec{a}_{j}=\sum_{k=j}^{m} \vec{\alpha}_{k}: j=1, \ldots, m$ as in (4), and thus $\vec{\tau}_{j}^{* \vec{c}} \leq|\vec{\alpha}|$ by (11)) we have

$$
\begin{aligned}
& \operatorname{deg} \mathbf{U}(z) \leq(n-1) \cdot d \\
& \operatorname{deg} \mathbf{T}(z) \leq|\vec{\alpha}|=\operatorname{deg} \operatorname{det} \mathbf{A}(z) \leq n \cdot d
\end{aligned}
$$

\section{Computing Popov Forms via Minimal Polyno- mial Bases}

In this section we show that shifted Popov forms $\mathbf{T}(z)$ of $\mathbf{A}(\Sigma)$ together with their multiplier $\mathbf{U}(z)$ can be obtained by a particular polynomial basis for the kernel of $\left[\mathbf{A}(z),-\mathbf{I}_{m}\right.$. (considered as a module over $\mathbb{Q}[z]$ ). It makes intuitive sense to look at the kernel since the columns of the $(m+n) \times n$ matrix polynomial

$$
\mathbf{S}(z)=\left[\begin{array}{c}
\mathbf{U}(z) \\
\mathbf{T}(z)
\end{array}\right]
$$

lic in the kernel of $\left[\mathbf{A}(z),-\mathbf{I}_{m}\right]$. These columns form a basis iff $\mathbf{T}(z)=\mathbf{A}(z) \cdot \mathbf{U}(z)$ and $\mathbf{U}(z)$ is unimodular. Finally, as observed for example in [19], we may use the algorithms FPHPS and SPHPS of [3] to compute column reduced polynomial bases of matrix polynomials, otherwise known as minimal polynomial bases (MPB). In our case we wish to use a shifted version of a basis of the kermel to compute shifted Popov forms.

\section{Definition 5.1 (Shifted Minimal Polynomial Bases)}

Let $\mathbf{C}(z) \in \mathbb{Q}^{m \times n}[z]$ be of rank $r$ and $\mathbf{B}(z) \in \mathbb{Q}^{n \times(n-r)}[z]$ irreducible (of full rank for all finite values of $z$ in the complex plane), with $\mathbf{C}(z) \cdot \mathbf{B}(z)=0$. If in addition $\mathbf{B}(z)$ is $\vec{b}$-column reduced then it is called $a \vec{b}$-Minimal Polynomial Basis $(\vec{b}-\mathrm{MPB})$ of the $\mathbb{Q}[\vec{z}]$-module kernel of $\mathbf{C}(z)$.

The existence and the uniqueness of a $\vec{b}$-MPB in $\vec{b}$-Popov form follows from Theorem 3.7. If $\vec{b}=0$ then Definition $\bar{j} .1$ gives the classical definition of a Minimal Polynomial Basis (MPB) [9]. Such bases are called minimal since if a MPB for the kernel of $\mathbf{A}(z)$ has degree $\vec{\beta}$ then any other basis has degree $\vec{\beta}^{\prime} \geq \operatorname{perm}(\vec{\beta})[12, \S 6.5 .4, \mathrm{p} 456]$.

\section{Theorem 5.2 (Popov forms via MPB)}

Let $\mathbf{A}(z)$ be an $m \times n$ matrix polynomial of full column rank, and $\vec{a}, \vec{c}$ multi-indices. Furthermore, let $\vec{\gamma}^{* \vec{c}} \geq$ $\operatorname{cdeg}\left(z^{-\vec{c}} \mathbf{A}(z)\right)$, and urite $\vec{n}(N)=\left(N \cdot \vec{e}-\vec{\gamma}^{\times \vec{c}} ; \vec{a}\right)$ for any integer $N$.

The matrix polynomial $\mathbf{S}(z)$ is of the form (13) with $\mathbf{T}(z)$ the $\vec{a}-$ Popov form of $\mathbf{A}(z)$ and $\mathbf{U}(z)$ its corresponding multiplier if and only if, for some

$$
N \geq N_{1}:=\Delta^{\vec{c}}+\max [\vec{a}-\vec{c}],
$$

$\mathbf{S}(z)$ is a MPB of the kernel of $\left[\mathbf{A}(z),-\mathbf{I}_{m}\right]$ in $\vec{n}(N)$-Popov form.

In this case, the latter property is true for all $N \geq N_{1}$. Furthermore, $\mathbf{S}(z)$ and $\mathbf{T}(z)$ have the same pivots, and the same shifted column degree.
Proof: For the first implication it is sufficient to show that the stacked matrix $\mathbf{S}(z)$ constructed with help of the Popov form $\mathbf{T}(z)$ and the multiplier $\mathbf{U}(z)$ is $\vec{n}(N)$-column reduced for all $N \geq N_{1}$, with the properties as specified in the last part of Theorem 5.2. Indeed, denoting by $\vec{x}$ the $\vec{a}$ column degree of $\mathbf{T}(z)$ and by $I$ the corresponding pivot set, we get.

$$
z^{-\vec{n}(N)} \cdot \mathbf{S}(z) \cdot z^{-\vec{a}+\vec{a}_{I}}=\left[\begin{array}{c}
z^{\vec{i}^{* \vec{e}}} \cdot \mathbf{U}(z) \cdot z^{-\vec{\alpha}+\vec{a}_{I}-N \cdot \vec{\varepsilon}} \\
z^{-\vec{a}} \cdot \mathbf{T}(z) \cdot z^{-\vec{a}+\vec{a}_{I}}
\end{array}\right],
$$

and it only remains to show that

$$
z^{\vec{\gamma}^{* \vec{c}}} \cdot \mathbf{U}(z) \cdot z^{-\vec{\alpha}+\vec{a}_{l}-N \cdot \vec{e}}=\mathcal{O}\left(z^{0}\right)_{z \rightarrow \infty}
$$

for all $N \geq N_{1}$. This latter statement follows however from (9) and (11).

In order to show the other implication, let $\mathbf{S}(z)$ (and $\widetilde{\mathbf{S}}(z)$ ) be as described in the first part of the assertion (and of the second part, respectively). Then the columns of both matrices form bases of the kernel of $\left[\mathbf{A}(z),-\mathbf{I}_{m}\right]$, and therefore there exists some unimodular $\mathbf{W}(z)$ such that $\widetilde{\mathbf{S}}(z)=\mathbf{S}(z) \cdot \mathbf{W}(z)$. On the other hand, both $\mathbf{S}(z)$ and $\tilde{\mathbf{S}}(z)$ are of full column rank and in $\vec{n}(N)$-Popov form for the specified value of $N$, and thus $\mathbf{S}(z)=\widetilde{\mathbf{S}}(z)$ by the uniqueness of $\vec{n}(N)$-Popov forms.

Theorem 5.2 implies that one can compute shifted forms by computing shifted forms of bases matrices for an associated kernel. That this is useful is shown by Theorem 5.3 below which implies that one can use the Mahler basis algorithm FFFG of [4] in order to compute an MPB $\mathbf{S}(z)$ in shifted Popov form. In addition, FFFG only uses fractionfree arithmetic, an advantage if the entries in the original matrix are polynomials having coefficients from an integral domain (for example a matrix with entries from $\mathbf{Z}[z]$ or $\left.\mathbb{Q}\left[a_{1}, \ldots, a_{k}\right][z]\right)$.

To bc more precise, let us briefly recall the definition of order for vector Hermite-Padé approximation (see, e.g., [5] or [4, Example 2.3]): given an $m \times s$ matrix $\mathbf{F}(z)$ of polynomials (or formal power series) and a multi-index $\vec{\sigma}$ of length $m$, a vector $\mathbf{P} \in \mathbb{Q}^{*}[z]$ is said to have order $\vec{\sigma}$ if $z^{-\vec{\sigma}} \cdot \mathbf{F}(z) \cdot \mathbf{P}(z)=\mathcal{O}(1)_{i \rightarrow 0}$. Obviously, the set of polynomial vectors having a given order is a submodule of $\mathbb{Q}^{s}[z]$, containing the kernel of $\mathbf{F}(z)$. The method FFFG mentioned above determines $s \times s$ bases of such order modules in shifted Popov form, by successively increasing one (arbitrary) component of the order vector by one unit. For sufficiently large order vectors and suitable shifts, we may recover our desired MPB in shifted Popov form as a part of such an order basis.

Theorem 5.3 Let $\mathbf{A}(z), \vec{a}, \vec{c}, \vec{\gamma}^{-\vec{c}}, \vec{n}$ be as in Theorem 5.2, and define $\vec{n}:=\left(N \cdot \vec{e}-\vec{\gamma}^{* \vec{c}}, \vec{a}\right)$, where $N \geq N_{1}$.

Apply algorithm FFFG to the malrix $\left[\mathbf{A}(z),-\mathbf{I}_{m}\right.$.] along the offdiagonal path induced by $\vec{n}$ and increasing order vectors $\vec{\sigma} \geq \overrightarrow{0}$ in order to obtain fraction-free polynomial bases $\mathbf{M}(z)$ of $\vec{n}$-column degree $\vec{\nu}$ along with polynomial residuals

$$
\mathbf{R}(z)=z^{-\vec{\sigma}} \cdot\left[\mathbf{A}(z),-\mathbf{I}_{m}\right] \cdot \mathbf{M}(z) \in \mathbb{Q}^{m \times(u+m)}[z],
$$

and stop the algarithm if a $m \times n$ submatrix $\mathbf{R}(z)_{+, L}$ of $\mathbf{R}(z)$ is zero.

Then there exists some scalar $c \neq 0$ such that the last $m$ rows of $\mathbf{M}(z)$. L give c times the $a-P$-Popov form of $\mathbf{A}(z)$ with 
$\vec{a}$-column degree $\vec{\alpha}=\vec{\nu}_{L}$ and pivot set $\left(\ell_{1}-n, \ldots, \ell_{n}-n\right)$, where $L=\left(\ell_{1}, \ldots, \ell_{n}\right)$, and the first n rous of $\mathbf{M}(z)_{*, L}$ give $c$ times the corresponding unimodular multiplier.

The algorithm will terminate at the latest by the order vector

$$
\vec{\sigma} \geq \vec{\sigma}^{\jmath}:=\vec{c}+\left(N+1+\max \left[\vec{\gamma}^{* \cdot \vec{\sigma}}\right]\right) \vec{e} .
$$

Proof: Notice that, for any order vector $\vec{\sigma}, \mathbf{R}(0)$ is of full rank $m[5$, Lemma 2.8], and $\mathbf{M}(z) / c$ is a nonsingular matrix in $\vec{n}$-Popov form for somc scalar $c \neq 0$ [4, Theorem 7.2], with its degree vector denoted by $\vec{\nu}$. Let $\mathbf{S}(z)$ be as in the first part of Theorem 5.2. Then the columns of $\mathbf{S}(z)$ have order $\vec{\sigma}$, and from $[4$, Theorem $7.3(\mathrm{a})]$ we know that there exists a unique matrix polynomial $\mathrm{P}(z)$ such that

$\mathbf{S}(z)=\mathbf{M}(\tilde{z}) \mathbf{P}(z), \quad$ and $\quad z^{\vec{\nu}-\vec{n}} \cdot \mathbf{P}(z) \cdot z^{-\vec{\alpha}+\vec{a}_{I}}=\mathcal{O}(1): \rightarrow \infty$

If the index set $L$ is as above, then the columns of $\mathbf{M}(z)_{*, L}$ are elements of the kernel of $\left[\mathbf{A}(z),-\mathbf{I}_{m}\right]$. From the basis property of $\mathbf{S}(z)$ we may conclude that there exists a unique matrix polynomial $\mathbf{Q}(z)$ such that $\mathbf{M}(z)_{*}, L=\mathbf{S}(z) \mathbf{Q}(\tilde{z})$. In combination with (14) we obtain $\mathbf{M}(z)_{*, L}=\mathbf{M}(z) \mathbf{P}(z) \mathbf{Q}(z)$. Consequently, $\mathbf{P}(z)_{L_{c}, *}=\mathbf{0}$, and $\mathbf{P}(z)_{L_{1},}=\mathbf{Q}(z)^{-1}$ is unimodular. Uniqueness of shifted Popov forms gives us the desired result. $\mathbf{S}(z)=\mathbf{M}(z))_{*, L} / c$.

In order to prove the last part, notice that

$$
\begin{aligned}
& z^{\vec{\sigma}-\vec{c}} \cdot \mathbf{R}(z) \cdot z^{\vec{n}-\vec{\nu}}=z^{-\vec{a}} \cdot\left[\mathbf{A}(z),-\mathbf{I}_{m}\right] \cdot \mathbf{M}(z) \cdot z^{\vec{n}-\vec{\nu}} \\
& =\left[z^{-\vec{c}} \cdot \mathbf{A}(z) \cdot z^{-\vec{\gamma}^{* \vec{c}}},-\mathbf{I}_{s}\right] \\
& \quad\left[\begin{array}{cc}
z^{N} \mathbf{I}_{n} & \mathbf{0} \\
\mathbf{0} & z^{\vec{a}-\vec{c}}
\end{array}\right] \cdot z^{-\vec{n}} \cdot \mathbf{M}(z) \cdot z^{\vec{n}-\overrightarrow{l^{\prime}}} \cdot
\end{aligned}
$$

Since $N-\max [\vec{a}-\vec{c}] \geq \Delta^{\vec{c}} \geq 0$ by assumption on $N$ and relation (10) of Theorem 4.1 , it follows that $z^{\vec{\sigma}-\vec{c}} \cdot \mathbf{R}(z)$. $z^{\vec{n}-\vec{v}-N \vec{e}}=\mathcal{O}\left(z^{0}\right)_{z \rightarrow \infty}$. We now may choose an index list $L$ with $L_{c}:=(1, \ldots, m+n) \backslash L$ such that the square matrix $\mathbf{R}(z)_{x_{i} L_{c}}$ is invertible. Consequently, there exists a bijective map $\rho:\{1, \ldots, m\} \rightarrow L_{c}$ with $\mathbf{R}(z)_{j, n(j)} \neq 0, j=1, \ldots, m$, and thus

$$
\operatorname{perm}(\vec{\sigma}-\vec{c}-N \vec{e}) \leq(\vec{\nu}-\vec{n})_{I_{c}}
$$

where we recall that perm denotes a permutation of the components. If now $\vec{\sigma} \geq \sigma^{f}=\vec{c}+N^{\prime} \cdot \vec{e}$, then min $\left[(\vec{\nu}-\vec{n})_{L_{e}}\right] \geq$ $N^{\prime}-N \geq \max \left[\vec{\gamma}^{*} \vec{a}\right]+1 \geq \max \left[\vec{\alpha}-\vec{a}_{I}\right]+1$ by assumption on $N^{\prime}$ and (12). It follows that $\mathbf{P}(z)_{L_{c_{1} *}}=\mathbf{0}$ in (14). On the other hand, with $\mathbf{S}(z), \mathbf{P}(z)$ must also have full column rank, and thus $\mathbf{P}(z)_{L, *}$ is invertible. Multiplying the lefthand equalion in (14) on the left with $\left[\mathbf{A}(z),-\mathbf{I}_{m}\right]$, we obtain $\mathbf{R}(z)_{*, L} \cdot \mathbf{P}(z)_{L, *}=\mathbf{0}$ and hence $\mathbf{R}(z)_{*, L}=\mathbf{0}$, as required for the final part of Theorem 5.3.

The fraction free computation of shifted Popov forms via FFFG has bcen implemented in the computer algebra system MAPLE and can be obtained from the authors web sites or via ermail.

Example 5.4 Let $\mathbf{A}(z)$ be as in Example 2.5 with $\vec{a}=\overrightarrow{0}$ and $\vec{c}=$ cdeg $\mathbf{A}(z)$. Then applying the FFFG algorithm along the path $[6,10,6,8,4,0]$ results in a Mahler system that produces an integer multiple of $a[8,4,0]$-Popov normal form $\mathbf{A}(z)$ along with a unimodular multiplicr in 18 itcrations. The result is 4 times the true ansuer because it works with fraction-free urithmelic. The unimodular multiplier in this case is given by

$$
\left[\begin{array}{ccc}
4 z^{2}+4 z & z+1 & -2 z+2 \\
-4 z^{2}+16 & -z & 2 z-4 \\
-4 z^{2}-8 z & -z-2 & 2 z
\end{array}\right]
$$

again 4 times the true answer.

We can give a worst case complexity for this approach in terms of $d=\operatorname{deg} \mathbf{A}(z)$. As in the discussion at the end of section 3 we will restrict ourselves to the choices $\vec{c}=0$ and $\vec{a} \geq 0, \max [\vec{a}] \leq|\vec{\alpha}|$, which includes the classical Popov and Hermite normal forms. Then $\vec{\gamma}^{*} \vec{a} \leq \vec{\gamma}^{0} \leq d \cdot \vec{e}$, and $\Delta^{\overrightarrow{0}}+\max [\vec{a}-\vec{c}] \leq\left|\vec{\gamma}^{* \overrightarrow{0}}\right| \leq n \cdot d$. Consequently, a practical choice for $N$ would be $N=n \cdot d$, leading to $\left|\vec{\sigma}^{f}\right|=m \cdot n \cdot d+$ $m \cdot(d+1)$.

From [5, Lemma 2.8] and [4, Section 4] we know that the module $K:=|\vec{\nu}|$ of the degree vector of the final basis in FFFG coincides with the module of the final order vector $|\vec{\sigma}| \leq\left|\vec{\sigma}^{f}\right|$. It is shown in $[3,4]$ that the complexity of FFFG to reach this order vector is bounded by $\mathcal{O}\left((m+n) \cdot K^{2}\right)$ in floating point arithmetic, and by $\mathcal{O}\left((m+n) \cdot \kappa^{2} \cdot K^{-4}\right)$ in exact arithmetic, where $\boldsymbol{k}$ is an upper bound for the size (in bits) of the coefficicnts of $\mathbf{A}(z)$ (remember that the corresponding complexities for solving the underlying systems of linear equations by Gaussian elimination are obtained by replacing $m+n$ above by $K$ ). Under the above assumptions, we obtain rough worst case bounds in terms of the degree $d=\operatorname{deg} A(z)$, namely $\mathcal{O}\left(m^{3} \cdot n^{2} \cdot d^{2}\right)$ floating point operations, and $\mathcal{O}\left(\kappa^{2} \cdot m^{5} \cdot n^{4} \cdot d^{4}\right)$ bit operations. There are no other fraction-free algorithms for Popov or Hermite that can be used for comparison with our approach.

\section{Conclusions}

In this paper we have studied reduced and normal forms of matrix polynomials by looking at so called shifted forms of matrix polynomials. Thesc forms include column-reduced, triangular, Hermite normal and Popov normal forms along with their shifted counterparts. We have determined degree bounds for a unimodular matrix which transforms the input matrix polynomial into an equivalent matrix in the desired form. The degree bounds allow one to embed a shifted Popov normal problem into a problem of determining a minimal polynomial basis in shifted Popov form for an associated stacked matrix polynomial. These shifted minimal polynomial bases can in turn be computed in a fraction-free way via the Mahler systom algorithm of [4].

As mentioned in the introduction the results in this paper can be viewed as a first step in a program to obtain efficient symbolic methods for computing matrix normal forms of arbitrary matrix polynomials. The full row rank rectangular case is important for computing matrix polynomial GCDs in normal form. The singular case gives information on minimal polynomial bases for the kernel of the matrix polynomial. The case of singular or rectangular matrices is considerably more complex because one no longer has a unique unimodular multiplier. In such cases one needs to determine a unimodular multiplier with minimal degree properties [6]. Degree bounds for this case are also more difficult. At the same time degree bounds for such multipliers in this case also lead to interesting degree bounds for important classes 
of problems of interest in computer algebra. For example, from [6] we have:

Theorem 6.1 (GCD of several scalar polynomials)

Let $\mathbf{A}(z)=\left[a_{1}(z), a_{2}(z), \ldots a_{n}(z)\right] \in \mathbb{Q}^{1 \times n}[z]$ with degrees $\vec{\gamma}=\left[\gamma_{1}, \gamma_{2}, \ldots \gamma_{n}\right]$ and $d(z)=G C D\left(a_{1}(z), \ldots, a_{n}(z)\right)$ with degree $\delta$. Assume (urithout loss of generality) that $\gamma_{1}=$ $\min _{j} \gamma_{j}$ and $\gamma_{n}=\max _{j} \gamma_{j}$. Then there are "small" multipliers $u_{k}(z)$ for the diophantine equation

$$
a_{1}(z) \cdot u_{1}(z)+a_{2}(z) \cdot u_{2}(z)+\ldots+a_{n}(z) \cdot u_{n}(z)=d(z),
$$

which satisfy

$$
\operatorname{deg} u_{1}(z) \leq \gamma_{k}-\delta-1, \quad \sum_{\substack{k=z \\ u_{k} \neq 0}}^{n}\left(1+\operatorname{deg} u_{k}(z)\right) \leq \gamma_{1}-\delta
$$

Notice that these bounds include the classical one for $n=$ 2 (cf. [10]). Also, a straight forward generalization of the integer bound of [11] to the polynomial case would lead to the weaker estimate deg $u_{k}(z) \leq \gamma_{n}-1$ for all $k$.

There are a number of interesting problems that still remain to be solved. We have shown that it is possible to solve the shifted Popov form problem via some fraction free algorithm by noting that it is embedded inside an order basis computation (or an MPB computation). The major problem with using such an approach to compute our form is that this method is not really a reduction procedure. In particular il does not recognize when a matrix polynomial $\mathbf{A}(z)$ is in shifted Popov form until the final step of the computation. We are interested in obtaining a fraction-free algorithm which computes minimal polynomial bases (and hence our normal form) in a reduction procedure. We expect that this may be done by determining an associated linear system along with determinental representations as in [4] and then making use of modified Schur complements as done in [1].

\section{References}

[1] Begkfrmain; B., Cabay, S., nND Labain, G. Fraction free computation of matrix padć systems. In Procecdings of ISSAC 97 (Maui, USA, 21-23 .July 1997), W. Küchlin, Ed., ACM Press, pp. 125-132.

[2] Beckermann, B.. and Labaix, G. A uniform approach for hermite padé and simultaneous padé approximants and their matrix generalizations. Numerical $\mathrm{Al}$ gorithms 3 (1992), 45-54.

[3] Beckermann, B., and Labahn, G. A uniform approach for the fast, reliable computation of matrix-type padé approximants. SIAM J. Matrix Anal. Appl. 15 (1994), 804823.

[4] Bechermann, B., axid Labahn, G. Fraction-free computation of matrix gcd's and rational interpolants. $S u b$ mitted to SIAM .J. Matrix Anal. Appl. (1997), 45pgs.

[5] BECkERMAN\%, B., ANI) LabaIIN, G. Recursiveness in matrix rational interpolation problems. J. Comput. Appl. Math. 77 (1997), 5-34

[6] Bechermann, B., Labahn, G., and Villard, G. Shifted normal forms of gencral polynomial matrices. manuscript. 1999.
[7] Beelex, T., van der Hurk, G., and Praagman, C. A new method for computing a column reduced polynomial matrix. Systems and Control Letters 10 (1988), $217-224$.

[8] BLltileel, A., and VA. Barel, M. A matrix euclidean algorithm and the matrix minimal padé approximation problem. Continued Fractions and Padé Approximants (1990).

[9] G.D. Forney, J. Minimal bases of rational vector spaces, with applications to multivariable linear systems. SIAM J. Control 13 (1975), 493520.

[10] Geddes, K., Czapor, S., and Labahn, G. Algorithrns for Computer Algebra. Kluwer, Boston, MA, 1992.

[11] Havas, G., Majewski, B., nvi Mattiews, K. Extended gcd and hermite normal form algorithms via lattice basis reduction. Experimental Mathematics 7(2) (1998), 125-135

[12] Kinifati, T. Linear Systems. Prentice-Hall, 1980.

[13] Kalionen, E., Krishnamoorthy, M., and SaunDERS, B. Parallel algorithms for matrix normal forms. Linear Algebra and its Applications 136 (1990), 189 208.

[14] Kannan, R. Solving systems of linear equations over polynomials. Theoretical Computer Science 39 (1985), 6988 .

[15] MacDuffef;, C. The Theory of Matrices. Chelsea, New-York, 1956.

[16] Newman, M. Integral Matrices. Academic Press, NewYork, 1972

[17] Popov, V. Some properties of control systems with irreducible matrix transfer functions. Lecture Notes in Mathematics 144 (1969), 169-180.

[18] Schrijver, A. Theory of Linear and Iruleger Programming. Wiley-Interscience series in Discrete Mathematics, 1986.

[19] Stuchlik-QuÉrF́, M. How to compute ninimal bases using padé approximants. Rapport de recherche lip6 1997/035, Laboratoire d'Informatique de Paris 6, France, 1997.

[20] Van Barel, M., AND Bultheel, A. A generalized minimal partial realization problem. Linear Algebra and its Applications 254 (1997), 527-551.

[21] Verghese, G., AND KAHATH, T. Rational matrix structure. IEEE Trans. Automat. Control 26 (1981), 434. 438 .

[22] Villard, G. Computing popov and hermite forms of polynomial matrices. In Proceedings of ISSAC 96 (Zurich, Switzerland, 24-26 July 1996), Y. Lakshman, Ed. ACM Press, pp. 250-258. 